\title{
DARTBOARD ARRANGEMENTS
}

\author{
G. L. Cohen* \\ School of Mathematical Sciences \\ University of Technology, Sydney \\ PO Box 123, Broadway \\ NSW 2007, Australia \\ glc@maths.uts.edu.au \\ E. Tonkes \\ School of Information Technology \\ Bond University \\ Gold Coast \\ Queensland 4229, Australia \\ etonkes@bond.edu.au
}

Submitted: February 17, 2000; Accepted: March 3, 2000.

AMS Subject Classification: Primary 05A05.

\begin{abstract}
This note considers possible arrangements of the sectors of a generalised dartboard. The sum of the $p$ th powers of the absolute differences of the numbers on adjacent sectors is introduced as a penalty cost function and a string reversal algorithm is used to determine all arrangements that maximise the penalty, for any $p \geq 1$. The maximum value of the penalty function for $p=1$ is well known in the literature, and has been previously stated without proof for $p=2$. We determine it also for $p=3$ and $p=4$.
\end{abstract}

\section{Introduction}

This note considers a combinatorial optimisation problem arising from the design of the standard dartboard, shown below. The arrangement may be construed as an effort to

${ }^{*}$ This paper was researched and prepared while a visitor at Bond University. The author is grateful for the hospitality shown by all. 
penalise a player's poor shots. By aiming for a large number (other than the inner bull worth 50 or the outer bull worth 25) but hitting a small number to one side of it, the penalty will be incurred. Is it possible for other arrangements to allow greater overall penalties?

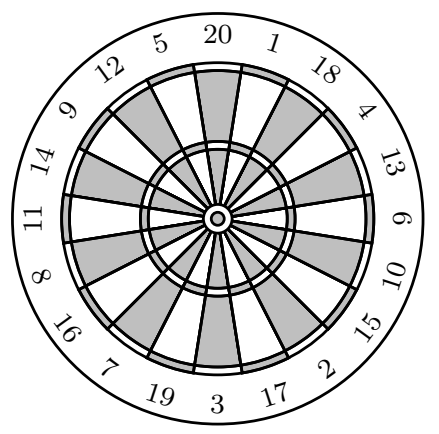

Several authors have considered this problem, sometimes generalised to a "dartboard" with $n$ sectors numbered $1,2, \ldots, n$, for $n \geq 3$, say. A philosophy of maximising some penalty function seems sensible, and two such functions have received most attention: the sum of the absolute differences of all pairs of adjacent numbers, and the sum of the squares of these differences. We shall refer to these criteria as defining the $L_{1}$ and $L_{2}$ problems, respectively.

Let $\mathcal{A}$ be the set of all permutations of $\{1,2, \ldots, n\}$ and write

$$
D_{p}(A)=\sum_{j=1}^{n}\left|i_{j}-i_{j+1}\right|^{p}
$$

where $A=\left(i_{1}, i_{2}, \ldots, i_{n}\right) \in \mathcal{A}$ and $i_{n+1}=i_{1}$. The problems may be given more generally as

$$
L_{p}: \quad \text { Find } \widehat{A} \in \mathcal{A} \text { such that } D_{p}(\widehat{A})=\max _{A \in \mathcal{A}} D_{p}(A) .
$$

For $p=1$ and $p=2$, the problem seems to have been discussed first by Selkirk [5]. He makes a number of assertions, all of which are correct but none of which are proved. The concentration of effort by the later writers suggests that the proofs are not as easy to obtain as Selkirk seems to imply. Eiselt and Laporte [2] formulate $L_{1}$ and $L_{2}$ in the context of a maximum-cost travelling salesman problem, and solve them using a branchand-bound algorithm. They only consider the case $n=20$. Everson and Bassom [3] give a direct solution of $L_{1}$, but do not consider $L_{2}$.

To our knowledge, the only other published discussion of the design problem is Lynch [4], who also related it to the travelling salesman problem. With $n=4 m$, for integer $m$, he took as his criterion that adjacent numbers be of opposite parity and have absolute differences greater than $2 m-2$.

Our intention here is to use a string reversal algorithm to solve $L_{p}$, for any $p \geq 1$.

We refer to the permutation $A$ above as a dartboard arrangement, and write it more usually as the string $A=i_{1} i_{2} \ldots i_{n}$. We call an arrangement $B \in \mathcal{A}$ equivalent 
to $A$ if $B=i_{q} i_{q+1} \ldots i_{n} i_{1} \ldots i_{q-1}$ for some $q, 1 \leq q \leq n$ (cyclic permutation of $A$ ), or $B=i_{r} i_{r-1} \ldots i_{1} i_{n} \ldots i_{r+1}$ for some $r, 1 \leq r \leq n$ (reversed cyclic permutation of $A$ ). For an actual dartboard, these imply that it does not matter which number is uppermost or whether the board is reflected in a diameter. In this paper, all arrangements are permutations in $\mathcal{A}$, except for a few references to the original dartboard arrangement, which we refer to as $A_{d}$.

We are particularly interested in alternating dartboard arrangements, for which we must distinguish between even and odd values of $n$. For $n=2 k$, let $\left(s_{1}, s_{2}, \ldots, s_{k}\right)$ be any permutation of $\{1,2, \ldots, k\}$, which we refer to as small numbers, and $\left(l_{1}, l_{2}, \ldots, l_{k}\right)$ be any permutation of $\{k+1, k+2, \ldots, 2 k\}$, which we refer to as large numbers. The string $s_{1} l_{1} s_{2} l_{2} s_{3} \ldots l_{k-1} s_{k} l_{k}$ of alternately small and large numbers is an alternating arrangement. For $n=2 k+1$, let $\left(s_{1}, s_{2}, \ldots, s_{k}\right)$ be any permutation of $\{1,2, \ldots, k\}$ and $\left(l_{1}, l_{2}, \ldots, l_{k}\right)$ be any permutation of $\{k+2, k+3, \ldots, 2 k+1\}$, and put $m=k+1$. The string $m s_{1} l_{1} s_{2} l_{2} s_{3} \ldots l_{k-1} s_{k} l_{k}$ is an alternating arrangement.

Notice that $A_{d}$ is not alternating.

The following result will be required.

Lemma 1 For any real numbers $a<b<c<d$, and any $p \geq 1$,

$$
(d-a)^{p}+(c-b)^{p} \geq(c-a)^{p}+(d-b)^{p},
$$

with equality if and only if $p=1$. Also

$$
\min \left\{(d-a)^{p}+(c-b)^{p},(c-a)^{p}+(d-b)^{p}\right\}>(d-c)^{p}+(b-a)^{p} .
$$

Proof: Put $\alpha=d-c, \beta=c-a$ and $\gamma=c-b$, so that $\alpha>0, \beta>\gamma>0$ and we are required to prove first that

$$
(\alpha+\beta)^{p}-\beta^{p} \geq(\alpha+\gamma)^{p}-\gamma^{p} .
$$

The function $f$, where $f(x)=(\alpha+x)^{p}-x^{p}$ for $x>0$, is easily seen to be increasing, and strictly so unless $p=1$. Then, for $p>1, f(\beta)>f(\gamma)$ as we wished to show.

Put $\delta=b-a$, so $\delta>0$. We must prove also that

$$
\min \left\{(\alpha+\gamma+\delta)^{p}+\gamma^{p},(\alpha+\gamma)^{p}+(\gamma+\delta)^{p}\right\}>\alpha^{p}+\delta^{p}
$$

But this formulation makes the result obvious.

\section{Solution of $L_{p}$}

We begin with

Lemma 2 For any $p \geq 1$, a necessary property of an arrangement $\widehat{A}$ that is a solution of $L_{p}$ is that $\widehat{A}$ is alternating. 
Proof: We will describe an algorithm that allows us to transform an arbitrary nonalternating arrangement $A^{\prime} \in \mathcal{A}$ into an alternating arrangement $A$ in such a manner that the penalty function is increased at each stage of the process. Then $D_{p}(A)>D_{p}\left(A^{\prime}\right)$, so a non-alternating arrangement could not be maximal. That will prove the theorem.

We assume first that $n$ is even: $n=2 k$. Notice in general that two large numbers appear successively in $A^{\prime}$ if and only if elsewhere two small numbers appear successively. The algorithm is as follows.

Step 1. From an arbitrary fixed number in the string move to the right, say. Identify the first occurrences of successive small numbers and successive large numbers. Reverse the substring from the second of the first pair to the first of the second pair, to give a new arrangement.

Step 2. If the result is an alternating arrangement, the process is complete. Otherwise, return to Step 1.

Following each application of Step 1, there are two fewer occurrences of successive small or large numbers, so the process must terminate in an alternating arrangement.

We will illustrate the algorithm with the string

$$
A_{1}=s_{1} l_{1} s_{2} l_{2} l_{3} l_{4} s_{3} l_{5} l_{6} s_{4} l_{7} s_{5} s_{6} s_{7} s_{8} l_{8} l_{9} s_{9}
$$

As this is written, moving to the right, the first occurrence of a large pair is $l_{2} l_{3}$ and the first occurrence of a small pair is $s_{5} s_{6}$. Applying the algorithm, we obtain the following sequence of strings, where the substrings to be reversed have been indicated within square brackets:

$$
\begin{aligned}
& A_{1}=s_{1} l_{1} s_{2} l_{2}\left[l_{3} l_{4} s_{3} l_{5} l_{6} s_{4} l_{7} s_{5}\right] s_{6} s_{7} s_{8} l_{8} l_{9} s_{9}, \\
& A_{2}=s_{1} l_{1} s_{2} l_{2} s_{5} l_{7} s_{4} l_{6}\left[l_{5} s_{3} l_{4} l_{3} s_{6}\right] s_{7} s_{8} l_{8} l_{9} s_{9}, \\
& A_{3}=s_{1} l_{1} s_{2} l_{2} s_{5} l_{7} s_{4} l_{6} s_{6} l_{3}\left[l_{4} s_{3} l_{5} s_{7}\right] s_{8} l_{8} l_{9} s_{9}, \\
& A_{4}=s_{1} l_{1} s_{2} l_{2} s_{5} l_{7} s_{4} l_{6} s_{6} l_{3} s_{7} l_{5} s_{3} l_{4} s_{8} l_{8}\left[l_{9} s_{9}\right], \\
& A_{5}=s_{1} l_{1} s_{2} l_{2} s_{5} l_{7} s_{4} l_{6} s_{6} l_{3} s_{7} l_{5} s_{3} l_{4} s_{8} l_{8} s_{9} l_{9},
\end{aligned}
$$

and $A_{5}$ is alternating. In $A_{4}$, note the implied pair $s_{9} s_{1}$.

At each repetition of Step 1, the value of $D_{p}$ is increased. To see this, consider the typical situation

$$
\begin{aligned}
& B=\ldots l_{1}\left[l_{2} \ldots s_{1}\right] s_{2} \ldots \\
& C=\ldots l_{1} s_{1} \ldots l_{2} s_{2} \ldots
\end{aligned}
$$

in which $C$ results from $B$ by application of Step 1. The calculations for the penalty function take the form

$$
\begin{aligned}
& D_{p}(B)=\Delta+\left|l_{1}-l_{2}\right|^{p}+\left|s_{1}-s_{2}\right|^{p}, \\
& D_{p}(C)=\Delta+\left(l_{1}-s_{1}\right)^{p}+\left(l_{2}-s_{2}\right)^{p},
\end{aligned}
$$


respectively, where $\Delta$ is precisely the same calculation in both sums. In each of the four possibilities, namely $s_{1}<s_{2}<l_{1}<l_{2}, s_{2}<s_{1}<l_{2}<l_{1}, s_{2}<s_{1}<l_{1}<l_{2}$ and $s_{1}<s_{2}<l_{2}<l_{1}$, the second part of Lemma 1 implies that

$$
\left|l_{1}-l_{2}\right|^{p}+\left|s_{1}-s_{2}\right|^{p}<\left(l_{1}-s_{1}\right)^{p}+\left(l_{2}-s_{2}\right)^{p} .
$$

Hence $D_{p}(B)<D_{p}(C)$, so we conclude that $D_{p}$ is maximal for an alternating arrangement.

When $n$ is odd, the median number is treated as a small number if it lies directly between two small numbers and as a large number if it lies directly between two large numbers. This of course includes situations such as the string $m s_{1} \ldots s_{2}$ (in which $m$ is treated as a small number). The algorithm may then be applied to arrive at an alternating arrangement, and the penalty function may be shown to increase, as before.

That completes the proof of Lemma 2.

We can now give a particular alternating arrangement that solves $L_{p}$. It is presented in a manner suggested by Brown [1].

Theorem 1 A solution of $L_{p}$ is given for each value of $n$ (mod 4) as follows. Reading each as a single string from left to right, we have

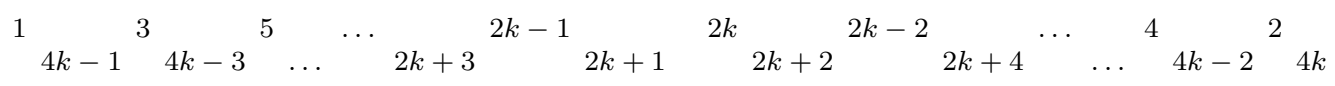

when $n=4 k$;

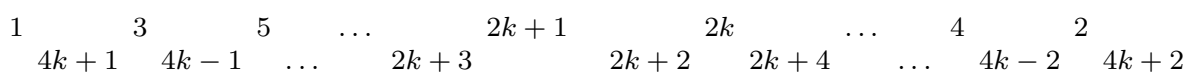

when $n=4 k+2 ;$ and

$$
{ }_{2 k}^{3}{ }^{3} 2 k-2^{5} \quad \cdots \quad{ }_{4}^{2 k-1} 2^{2 k+1}
$$

when $n=2 k+1$

Furthermore, this solution is unique, up to equivalence, when $p>1$.

Proof: The above strings may be given equivalently, for all $n$, as the first row of the following array:

$$
\begin{array}{cccccccccccc}
\ldots & 5 & n-3 & 3 & n-1 & 1 & n & 2 & n-2 & 4 & n-4 & \ldots \\
\ldots & 9 & 7 & 5 & 3 & 1 & 2 & 4 & 6 & 8 & 10 & \ldots
\end{array}
$$

Let this string be $A$, and let $A^{\prime \prime}$ be an arbitrary string in $\mathcal{A}$. By the algorithm in the proof of Lemma 2, we may transform $A^{\prime \prime}$ into an alternating string $A^{\prime}$, and increase the penalty function. The second row of the preceding array indicates the order in which we shall move the numbers of $A^{\prime}$ to transform it into $A$, again using string reversal. That is, we fix 1 in $A^{\prime}$, then move $n$ into place to the right of 1 , then $n-1$ to the left of 1 , then move 2 , then 3 , then $n-2, \ldots$ Lemma 1 will imply quickly that the penalty function $D_{p}$ is increased with each move, and that implies that $A$ is maximal.

To be specific, consider the intermediate string

$$
\begin{array}{llllllllllllllllll}
\ldots & l & k & \ldots & s & n-k+2 & k-2 & \ldots & 5 & n-3 & 3 & n-1 & 1 & n & 2 & n-2 & 4 & \ldots
\end{array}
$$


where it is required now to move the small number $k$ into place. As before, $l$ denotes a large number and $s$ a small number. Let

$$
\begin{aligned}
& B=\ldots l\left[\begin{array}{lll}
k & \ldots & s
\end{array}\right] n-k+2 \ldots \\
& C=\ldots l \quad s \ldots k n-k+2 \ldots \text {, }
\end{aligned}
$$

where the substring $k \ldots s$ in $B$ has been reversed in $C$. Observe that $k<s<l<$ $n-k+2$, by virtue of the order in which the transformation is taking place. By the first part of Lemma 1,

$$
(n-2 k+2)^{p}+(l-s)^{p} \geq(l-k)^{p}+(n-k+2-s)^{p},
$$

so that $D_{p}(B) \leq D_{p}(C)$, with strict inequality if $p>1$. The situation is precisely similar if $k$ is now a large number to be moved into place, as from

$$
\ldots s[k \ldots l] n-k k+2 \ldots \text { to } \ldots . s \quad l \ldots k n-k k+2 \ldots,
$$

where $n-k<s<l<k$. As written, these two cases suggest that $k$ is odd, but the situation is again precisely similar if $k$ is even.

The conclusion follows, as indicated above, and, except for equivalent arrangements, the solution will be unique if $p>1$.

\section{Further remarks}

(1) For $L_{1}$, we can prove that an alternating arrangement is also sufficient for a solution. Our method is different from that of Everson and Bassom [3].

Theorem 2 A dartboard arrangement in $\mathcal{A}$ is a solution of $L_{1}$ if and only if it is alternating.

Proof: By Lemma 2, for the proof we need only show that the penalty function $D_{1}(A)$ is the same for any alternating arrangement $A \in \mathcal{A}$.

Suppose $n$ is even, $n=2 k$. For $A=s_{1} l_{1} s_{2} l_{2} s_{3} \ldots l_{k-1} s_{k} l_{k}$, we have

$$
\begin{aligned}
D_{1}(A) & =\left(l_{1}-s_{1}\right)+\left(l_{1}-s_{2}\right)+\left(l_{2}-s_{2}\right)+\cdots+\left(l_{k}-s_{k}\right)+\left(l_{k}-s_{1}\right) \\
& =2\left(l_{1}+l_{2}+\cdots+l_{k}\right)-2\left(s_{1}+s_{2}+\cdots+s_{k}\right) \\
& =2(((k+1)-1)+((k+2)-2)+((k+3)-3)+\cdots+(2 k-k)) \\
& =2 k^{2} .
\end{aligned}
$$

For $n$ odd, $n=2 k+1$, and $A=m s_{1} l_{1} s_{2} l_{2} s_{3} \ldots l_{k-1} s_{k} l_{k}$, it is easy to see that, again, $D_{1}(A)=2 k^{2}$.

In summary, for every alternating arrangement $A \in \mathcal{A}, D_{1}(A)=2[n / 2]^{2}$, where $[\cdot]$ is the greatest integer function. 
Notice that $2[n / 2]^{2}=200$ when $n=20$, whereas $D_{1}\left(A_{d}\right)=198$.

(2) Selkirk [5] gives the result

$$
D_{2}(\widehat{A})= \begin{cases}\frac{1}{3} n^{3}-\frac{4}{3} n+2, & n \text { even } \\ \frac{1}{3} n^{3}-\frac{4}{3} n+1, & n \text { odd }\end{cases}
$$

which may be confirmed from the maximal arrangements given in Theorem 1 .

When $n=20$, we have $D_{2}(\widehat{A})=2642$, whereas $D_{2}\left(A_{d}\right)=2374$.

We have also obtained the following results:

$$
\begin{aligned}
& D_{3}(\widehat{A})= \begin{cases}\frac{1}{4} n^{4}-2 n^{2}+3 n, & n \text { even, } \\
{[2] \frac{1}{4} n^{4}-2 n^{2}+3 n-\frac{5}{4},} & n \text { odd },\end{cases} \\
& D_{4}(\widehat{A})= \begin{cases}\frac{1}{5} n^{5}-\frac{8}{3} n^{3}+6 n^{2}-\frac{68}{15} n+2, & n \text { even }, \\
{[2] \frac{1}{5} n^{5}-\frac{8}{3} n^{3}+6 n^{2}-\frac{68}{15} n+1,} & n \text { odd } .\end{cases}
\end{aligned}
$$

(3) A different criterion has been proposed by Brown [1]. He suggests minimising $\sum_{j=1}^{n}\left(i_{j}+\right.$ $\left.i_{j+1}\right)^{2}$, in the notation of our introduction. Since

$$
\sum_{j=1}^{n}\left(i_{j}+i_{j+1}\right)^{2}=\sum_{j=1}^{n} i_{j}^{2}+\sum_{j=1}^{n} i_{j+1}^{2}+2 \sum_{j=1}^{n} i_{j} i_{j+1}=2 \sum_{j=1}^{n} j^{2}+2 \sum_{j=1}^{n} i_{j} i_{j+1}
$$

and

$$
\sum_{j=1}^{n}\left(i_{j}-i_{j+1}\right)^{2}=2 \sum_{j=1}^{n} j^{2}-2 \sum_{j=1}^{n} i_{j} i_{j+1}
$$

we have

$$
\sum_{j=1}^{n}\left(i_{j}+i_{j+1}\right)^{2}=\frac{2}{3} n(n+1)(2 n+1)-\sum_{j=1}^{n}\left(i_{j}-i_{j+1}\right)^{2}
$$

so Brown's approach is seen to be equivalent to the problem $L_{2}$.

(4) It is apparent that our methods rely on the ordering of the natural numbers. They may be applied equally to any set of real numbers: rank the numbers in increasing order, with equal numbers given successive increasing ranks arbitrarily. Then Theorem 1, applied to the rankings and then interpreted in terms of the original numbers, will give an arrangement that solves the analogue of $L_{p}$, and, for $p>1$, the solution will be unique up to equivalence.

(5) In a private communication, Dr Neil Williams has pointed out to us that string reversal has been used on a related problem in the 1983 Australian Mathematical Olympiad. We are grateful for that comment, and also for referring us to the article by Lynch [4]. 


\section{References}

[1] K. S. Brown, "The dartboard sequence", downloaded from the website http://www.seanet.com/ ${ }^{\sim}$ ksbrown/kmath025.htm (posted February 1996).

[2] H. A. Eiselt and G. Laporte, "A combinatorial optimization problem arising in dartboard design", J. Op. Res. Soc., 42 (1991), 113-118.

[3] P. J. Everson and A. P. Bassom, "Optimal arrangements for a dartboard", Math. Spectrum, 27 (1994/5), 32-34.

[4] M. A. M. Lynch, "Designing a dartboard - an application of graph modelling", Teaching Math. and Its Appl., 16 (1997), 51-54.

[5] K. Selkirk, "Re-designing the dartboard", Math. Gaz., 60 (1976), 171-178. 